\title{
Phase Transitions in a Transverse Ising Film with the Next Nearest-Neighbor Interactions
}

\author{
Li Zhi-Peng ${ }^{1, \text { a }}$ \\ ${ }^{1}$ School of Automobile Engineering, Linyi University, Linyi 276005, China \\ aemail: lizhipeng@lyu.edu.cn
}

Keywords: ferroelectrics; transverse Ising film; Curie temperature

Abstract. Using the mean-field approximation, we employ the transverse Ising model with the next nearest-neighbor interactions to investigate the phase transitions in a ferroelectric thin film. The dependence of the Curie temperature on the transverse field, the surface exchange interaction and the thin film thickness are calculated. The next nearest-neighbor interaction is supposed to decay with the distance between the pseudo-spins as a power law.

\section{Introduction}

Ferroelectrics have been receiving considerable concern for various potential applications experimentally and theoretically [1]. In particular, the surface and size effects on phase transitions in ferroelectrics have aroused renewed interest recently with the development of fabrication technology in ferroelectric thin films. In theory, the transverse Ising model is thought to be an excellent method to study the ferroelectric thin film from the view of microcosmic point [2-9]. Wang et al. studied the influence of the surface exchange interaction and the surface film thickness as well as the total film thickness on the Curie temperature of ferroelectric films described by the transverse Ising model with the mean-field approximation [2]. Sy introduced the surface modification to ferroelectric films and expressed the Curie temperature in terms of general determinants [4]. In these literatures, only the nearest-neighbor interactions are considered and the next nearest-neighbor interactions are neglected. Realistically, the long-range interactions dominate in ferroelectrics so that the next nearest-neighbor interactions should be included. At the beginning, Wang et al. investigated the polarization and the Curie temperature of ferroelectric films by considering different ranges of exchange interactions [5]. Subsequently, Xin et al. extended the theory to ferroelectric square lattices and obtained some valuable results [6]. Within the framework of the effective-field theory with correlations, Yao et al. [7] and Tabyaoui et al. [8] also calculated the dependence of Curie temperature on various parameters, respectively. As mentioned above, however, there are still very little works on the next nearest-neighbor interaction.

In this present work, the transverse Ising model is used to study the phase transitions in ferroelectric thin films with the next nearest-neighbor interactions by the mean-field approximation. It is assumed that the next nearest-neighbor interaction can decay with the distance between the pseudo-spins as a power law. The numerical results for the dependence of the Curie temperature on the transverse field, the surface exchange interaction and the film thickness are obtained.

\section{Model and formulas}

The structure of the transverse Ising film is shown in Fig. 1. The Hamiltonian is [5-9]

$$
H=-\frac{1}{2} \sum_{i j} \frac{J_{i j}}{r_{i j}^{\delta}} S_{i}^{Z} S_{j}^{Z}-\Omega_{b} \sum_{i \in b} S_{i}^{X}-\Omega_{s} \sum_{i \in S} S_{i}^{X},
$$

where $\mathrm{S}_{\mathrm{i}}{ }^{\mathrm{x}}$ (or $\mathrm{S}_{\mathrm{i}}^{\mathrm{z}}$ ) is the spin- $1 / 2$ component in the direction of $\mathrm{x}$ (or $\mathrm{z}$ ) at the site $\mathrm{i} . \Omega_{\mathrm{i}}$ is the transverse field in the $\mathrm{i}$-th layer. The transverse field in the surface layer is $\Omega_{\mathrm{i}}=\Omega_{\mathrm{s}}$, while the transverse field in 
the bulk layer is $\Omega_{\mathrm{i}}=\Omega_{\mathrm{b}}$. $\mathrm{J}_{\mathrm{ij}}$ is the exchange interaction between two pseudo-spins. $\delta$ is the decaying exponent.

According to the mean-field approximation $[5,6]$, the polarization in the $\mathrm{i}$-th layer is

$$
R_{i}=\left\langle S_{i z}\right\rangle=\left(\left\langle H_{i z}\right\rangle / 2\left|H_{i}\right|\right) / \tanh \left(\left|H_{i}\right| / 2 k_{B} T\right),
$$

where

$$
\begin{aligned}
& \left\langle H_{i z}\right\rangle=\sum_{j} \frac{J_{i j}}{r_{i j}^{\delta}} S_{j}^{z}, \\
& \left|H_{i}\right|=\sqrt{\Omega_{i}^{2}+\left\langle H_{i z}\right\rangle^{2}} .
\end{aligned}
$$

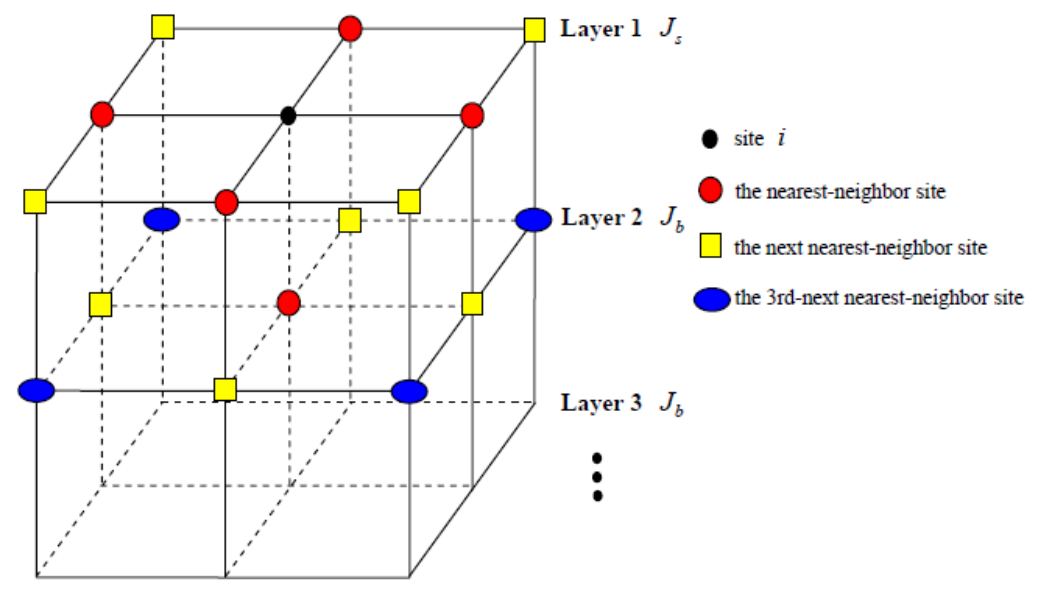

Fig. 1 The structure diagram of the transverse Ising film.

For the transverse Ising film with a thickness of $n$, the Eq. 1 will be extended to $n$ linear equations. For the top surface layer $(\mathrm{i}=1)$, there is

$$
\tau_{s} R_{1}=4 J_{s} R_{1}+J_{b} R_{2}+4 \frac{J_{s}}{\sqrt{2}^{\delta}} R_{1}+4 \frac{J_{b}}{\sqrt{2}^{\delta}} R_{2} .
$$

For the bulk layer (or the i-th layer), there is

$$
\tau_{b} R_{i}=4 J_{b} R_{i}+J_{b} R_{i-1}+J_{b} R_{i+1}+4 \frac{J_{b}}{\sqrt{2}^{\delta}} R_{i}+4 \frac{J_{b}}{\sqrt{2}^{\delta}} R_{i-1}+4 \frac{J_{b}}{\sqrt{2}^{\delta}} R_{i+1} .
$$

For the bottom surface layer $(\mathrm{i}=\mathrm{N})$, there is

$$
\tau_{s} R_{N}=4 J_{s} R_{N}+J_{b} R_{N-1}+4 \frac{J_{s}}{\sqrt{2}^{\delta}} R_{N}+4 \frac{J_{b}}{\sqrt{2}^{\delta}} R_{N-1} .
$$

Where

$$
\begin{gathered}
\tau_{s}=2 \Omega_{s} \operatorname{coth}\left(\frac{\Omega_{s}}{2 k_{B} T}\right), \\
\tau_{b}=2 \Omega_{b} \operatorname{coth}\left(\frac{\Omega_{b}}{2 k_{B} T}\right) .
\end{gathered}
$$

When the coefficient determinant of $\mathrm{R}_{\mathrm{i}}$ is equal to zero, the Curie temperature of the transverse Ising film can be obtained as 


$$
\left|\begin{array}{ccccccc}
X_{s} & -1 & & & & & \\
-1 & X_{b} & -1 & & & & \\
& -1 & X_{b} & & & & \\
& & & \ddots & & & \\
& & & & X_{b} & -1 & \\
& & & & -1 & X_{b} & -1 \\
& & & & & -1 & X_{s}
\end{array}\right|_{N \times N}=0
$$

where

$$
X_{s}=\frac{\tau_{s}-4 J_{s}-4 \frac{J_{s}}{\sqrt{2}^{\delta}}}{J_{b}+4 \frac{J_{b}}{\sqrt{2}^{\delta}}}, X_{b}=\frac{\tau_{b}-4 J_{b}-4 \frac{J_{b}}{\sqrt{2}^{\delta}}}{J_{b}+4 \frac{J_{b}}{\sqrt{2}^{\delta}}} .
$$

\section{Numerical results}

Employing Eq. (10), phase transition behaviors of the transverse Ising film with next nearest-neighbor interaction can be calculated. For simplicity, it is assumed that $\Omega_{\mathrm{s}}=\Omega_{\mathrm{b}}=\Omega$.
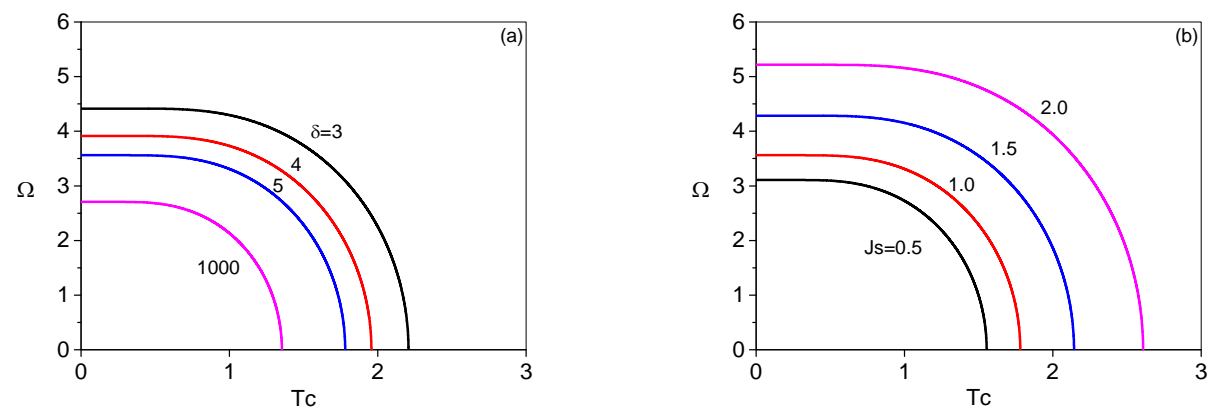

Fig. 2 The dependence of Curie temperature Tc on the transverse field $\Omega$.
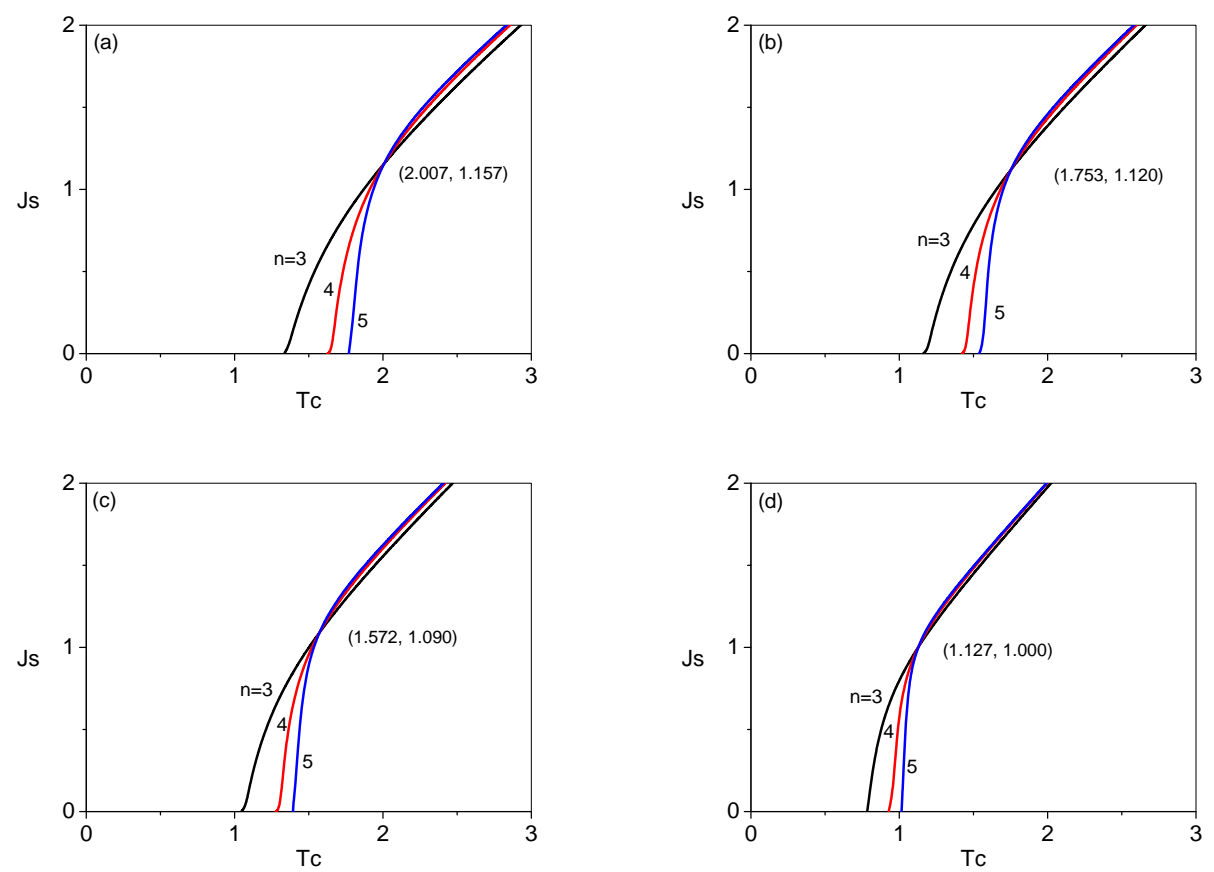

Fig. 3 The dependence of Curie temperature Tc on the surface interaction Js for different $n$. 
Fig. 2 shows the dependence of Curie temperature Tc on the transverse field $\Omega$ for a transverse Ising film with thickness of $n=3$. In Fig. 2(a), Js $=J b=1.0$ is considered. From Fig. 2(a), it can be found that the Curie temperature is increased with the transverse field decreases. But for the different decaying exponent $\delta$, the critical Curie temperature is decreased with the value of $\delta$ increases. Fig. 2(b) also shows the variation of Curie temperature on the transverse field for four different interactions Js when $\delta=5.0$. However, it is observed that the critical Curie temperature is increased with the value of Js increases.

Fig. 3 shows the dependence of Curie temperature Tc on the surface interaction Js for different decaying exponent $\delta$ and film thickness n. From Figs. 3(a)-(d), the value of $\delta$ is 3, 4, 5, 1000, respectively. Firstly, it is obvious that the curves of Js $\sim \mathrm{Tc}$ intersect at a common point. Moreover, the common point is independent on the film thickness $\mathrm{n}$. On the left side of common point, the Curie temperature Tc increases as the film thickness $n$ increases. But on the right side of common point, the Curie temperature Tc decreases as the film thickness $n$ increases. The coordinates for different $\delta$ of 3 , $4,5,1000$ are $(2.007,10157),(1.753,10120),(1.572,1.090),(1.127,1.000)$ as shown in Figs. (a)-(d). Obviously, the coordinate values of Js and Tc are both decreased with the decaying exponent $\delta$ increases. In fact, the decaying exponent $\delta=1000$ in Fig. 3(d) corresponds to the short-range interaction.

\section{Conclusions}

By taking into account the next nearest-neighbor interaction, the phase diagrams of a transverse Ising film have been briefly checked. The impact of the transverse field on the Curie temperature at different decaying exponent $\delta$ and surface interaction is discussed. Meanwhile, the impact of the surface interaction on the Curie temperature is also calculated. The numerical results indicate that the next nearest-neighbor exchange interaction for different decaying exponent $\delta$ and the transverse field as well as the film thickness all have a significant influence on the phase transition features of the transverse Ising film.

\section{References}

[1] J.F. Scott: Science Vol. 315 (2007), p. 954

[2] C.L. Wang, W.L. Zhong and P.L. Zhang: J. Phys.: Condens. Matter Vol. 4 (1992), p. 4743

[3] C.L. Wang, S.R.P. Smith and D.R. Tilley: J. Phys.: Condens. Matter Vol. 6 (1994), p. 9633

[4] H.K. Sy: J. Phys.: Condens. Matter Vol. 5 (1993), p. 1213

[5] Y.G. Wang, W.L. Zhong and P.L. Zhang: Solid State Commun. Vol. 101 (1997), p. 807

[6] Y. Xin, C.L. Wang, W.L. Zhong and P.L. Zhang: Phys. Lett. A Vol. 260 (1999), p. 411

[7] D.L. Yao, Y.Z. Wu and Z.Y. Li: Phys. Status Solidi B Vol. 231 (2002), p. 3

[8] A. Tabyaoui, M. Madani, A. Ainane and M. Saber: Physica A Vol. 358 (2005), p. 150

[9] Z.P. Li: submitted to Phase Transitions (2015) 